\title{
Jackson-Pratt drainage in pediatric craniofacial reconstructive surgery: is it helping or hurting?
}

\author{
Kumar Vasudevan, MD, ${ }^{1,2}$ Ahyuda Oh, DDS, MBA, DrPH, ${ }^{3}$ R. Shane Tubbs, PhD, PA-C, ${ }^{4}$ \\ David Garcia, MD, ${ }^{1,2}$ Andrew Reisner, MD, ${ }^{1,2}$ and Joshua J. Chern, MD, PhD ${ }^{1,2}$ \\ ${ }^{1}$ Pediatric Neurosurgery Associates, Children's Healthcare of Atlanta; ${ }^{2}$ Department of Neurosurgery, Emory University; \\ ${ }^{3}$ Department of Pediatrics, Emory University School of Medicine, Atlanta, Georgia; and ${ }^{4}$ Seattle Science Foundation, \\ Seattle, Washington
}

OBJECTIVE Jackson-Pratt drains (JPDs) are commonly employed in pediatric craniofacial reconstructive surgery (CRFS) to reduce postoperative wound complications, but their risk profile remains unknown. Perioperative blood loss and volume shifts are major risks of CFRS. The goal of this study was to evaluate the risks of JPD usage in CFRS, particularly with regard to perioperative blood loss, hyponatremia, intensive care unit (ICU) length of stay, and postoperative wound complications.

METHODS The authors performed a retrospective review of data obtained in pediatric patients who underwent CFRS at a single institution, as performed by multiple surgeons between January 2010 and December 2014. Data were gathered from patients who did and did not receive JPDs at the time of surgery. Outcome measures were compared between the JPD and no-JPD groups.

RESULTS The overall population 179 pediatric patients: 128 who received JPDs and 51 who did not. In their analysis, the authors found no significant differences in baseline patient characteristics between the two groups. The average JPD output over the first 48 hours was $222 \pm 142 \mathrm{ml}$. When examining the immediate preoperative to immediate postoperative time period, no significant differences were noted between the groups with regard to the need for blood transfusion or changes in hemoglobin, hematocrit, or serum sodium levels. These differences were also not significant when examining the 48-hour postoperative period. Finally, no significant differences in hospital length of stay, ICU length of stay, or emergency department visits at 60 days were noted between the two groups.

CONCLUSIONS In this retrospective study, the use of JPDs in pediatric CFRS was not associated with an increased risk of serious perioperative complications, although the benefits of this practice remain unclear.

https://thejns.org/doi/abs/10.3171/2017.5.PEDS17101

KEY WORDS craniofacial surgery; craniosynostosis; surgical complications; Jackson-Pratt drain

$\mathrm{C}$ RANIOFACIAL reconstructive surgery (CFRS) is commonly employed to correct pediatric craniosynostosis, the congenital premature ossification of calvarial sutures that alters the normal growth pattern of the skull. ${ }^{5}$ To optimize calvarial development, these procedures are usually performed on infants between the ages of 3 and 8 months, although occasional reconstructions are performed on children up to $4-5$ years old. ${ }^{2}$ These proce- dures can be associated with acquired coagulopathy, largevolume blood loss, and marked volume shifts during the perioperative period. Allogenic blood transfusion is the most common health care procedure in the United States, ${ }^{8}$ and in children it carries both infectious and noninfectious risks such as coagulopathy, overtransfusion, transfusionassociated acute lung injury, hemolysis, and anaphylaxis. ${ }^{10}$ There is a need for systematic examination of the factors

ABBREVIATIONS ADH = antidiuretic hormone; CFRS = craniofacial reconstructive surgery; $E B L=$ estimated blood loss; ICU = intensive care unit; JPD = Jackson-Pratt drain; $P O D=$ postoperative day.

SUBMITTED February 28, 2017. ACCEPTED May 1, 2017.

INCLUDE WHEN CITING Published online July 21, 2017; DOI: 10.3171/2017.5.PEDS17101. 
contributing to the perioperative blood loss and other morbidities seen in pediatric CFRS.

A growing multidisciplinary body of work has sought to minimize the risks associated with CFRS by improving hemostasis, exploring minimally invasive procedures, utilizing blood-sparing anesthetic and resuscitation algorithms, and addressing factors in postoperative care that can contribute to blood loss and the need for transfusion. The use of surgical drains, in particular with prophylactic closed suction drainage, has been implicated in a number of postoperative surgical complications, although studies of the routine placement of these drains have yielded inconsistent results. ${ }^{3}$ The Jackson-Pratt drain (JPD), placed in the subgaleal space with closed suction drainage, consists of a soft internal drain component connected by flexible tubing to a grenade-shaped bulb that is "charged" by squeezing air out to create a directional air pressure gradient, drawing blood and other fluids from the operative bed into the bulb. This provides continuous, low-pressure drainage in a portable system that lies as close as possible to the operative site.

JPDs typically function to close the potential space created by surgical dissection by preventing blood and fluid accumulation that could contribute to wound dehiscence or infectious or neurological complications. Patients with reduced surgical site edema also benefit from earlier discharge and increased comfort; this is especially important in infant patients undergoing CFRS who routinely have difficulty opening their eyes owing to periorbital edema and who experience significant shifting of subgaleal fluid collections with changes of position. However, the benefits of JPD placement in any of a number of operative procedures, including craniotomy, have never been reliably demonstrated, ${ }^{1}$ and recommendations regarding JPD usage remain uncertain.

For instance, Choi et al., in a retrospective study assessing pterional craniotomy both with and without JPD placement, found that JPD placement led to a thinner myocutaneous flap postoperatively (this was interpreted as reduced postoperative hematoma size). However, this was accompanied by a significant increase in the number of postoperative epidural hematomas in the JPD group. Rates of infection did not differ significantly between the two groups. While the radiographic methods used in their study are certainly prone to error, the authors concluded that JPD placement "does not appear to be necessary for prevention of postoperative surgical site hematoma as well as for promotion of surgical site healing in pterional craniotomy." Similarly, Van Roost et al. ${ }^{12}$ described a new phenomenon of "pseudohypoxic brain swelling" whereby 17 postcraniotomy patients displayed bilateral fulminant cerebral edema, mostly in the basal ganglia and thalami. After further investigation (including brain biopsy) ruled out other causes, closed suction drainage was ultimately thought to be the causative factor. Perhaps most concerning are numerous reports ${ }^{4,6}$ of bradyarrhythmias and cardiac arrest, some noted intraoperatively, after the closed suction drains were applied beneath a craniotomy flap. However, these and other serious complications have not been demonstrated prospectively or in larger retrospective series.
In this retrospective review study, we sought to determine possible risks from JPD placement in CFRS patients, particularly regarding the feared complication of perioperative blood loss. We first determined the degree of perioperative blood loss for all patients, as measured by perioperative hemoglobin and hematocrit changes and the need for transfusion. We then sought to understand whether JPD usage was associated with further blood loss or development of hyponatremia during the postoperative period. Last, we examined the potential effect of JPDs on length of intensive care unit (ICU) or hospital stay and subsequent wound complications. We hypothesized that the use of JPDs would be associated with greater postoperative blood loss, that patients who received a JPD would have a drop in serum sodium (based on prior studies that described release of ADH in these patients), and that increased drainage through the JPD would be associated with a greater postoperative blood loss. We did not expect that the presence of a JPD would be associated with increased length of stay in the ICU or in the hospital overall, given that in our experience JPDs are usually discontinued prior to when most patients are stable for discharge. Finally, we hypothesized that the use of JPDs would be associated with a greater number of wound complications (including infections) because JPD exit sites are typically close to wound edges and might allow for infection or wound breakdown.

\section{Methods}

\section{Patient Selection}

We conducted a retrospective review of CFRS cases at Children's Healthcare of Atlanta over the course of 5 years (January 2010-December 2014). For this study, procedures were included if they involved the following: 1) bicoronal incisions, 2) craniectomy as a portion of the procedure, 3) craniofacial reconstruction with or without implanted hardware, and 4) craniosynostosis as a diagnosis. All surgeries were performed by pediatric neurosurgeons $(n=3)$ collaborating with pediatric plastic craniofacial surgeons $(\mathrm{n}=2)$. Cases involving a "release" of sutures or any reoperations were excluded. Endoscopic procedures were also excluded. To limit the study to children with comparable physiology, we excluded all patients older than 24 months at the time of surgery. No cases were excluded on the basis of number or location of sutures being treated. Patients with syndromic craniosynostosis were included. Each patient was tracked for 60 days after time of discharge. After excluding those patients without a complete set of laboratory information (see below), 179 patients were included in this study.

All patients were considered for a JPD at the time of surgery. When used, all JPDs were placed in the subgaleal space just prior to closure and tunneled out through a remote stab incision. The decision to use a JPD was made intraoperatively by the treating surgeons on an individual basis. In accordance with our institution's standard protocols, all patients received perioperative antibiotic prophylaxis in the form of weight-adjusted doses of cefazolin or vancomycin. All antibiotics were discontinued at 24 hours postoperatively, independent of JPD status. Postopera- 
TABLE 1. Patient characteristics of JPD and no-JPD groups

\begin{tabular}{|c|c|c|c|c|}
\hline \multirow[b]{2}{*}{ Characteristic } & \multirow{2}{*}{$\begin{array}{c}\text { All Patients } \\
(\%)\end{array}$} & \multicolumn{2}{|c|}{ Group (\%) } & \multirow{2}{*}{$\begin{array}{c}p \\
\text { Value }\end{array}$} \\
\hline & & JPD & No JPD & \\
\hline No. of patients & 179 & 128 & 51 & \\
\hline Mean age $\pm S D$, mos & $8.67 \pm 4.75$ & $9.03 \pm 3.99$ & $8.32 \pm 3.76$ & 0.44 \\
\hline Sex, F/M & $82: 97$ & $53: 75$ & $29: 22$ & \\
\hline \multicolumn{5}{|l|}{ Diagnosis, no. of patients } \\
\hline Sagittal & $72(40.2)$ & $50(37.5)$ & $22(43.1)$ & 0.39 \\
\hline Metopic & $39(21.8)$ & $30(23.4)$ & $9(17.6)$ & 0.21 \\
\hline Unicoronal & $24(13.4)$ & $16(8.9)$ & $8(15.7)$ & 0.19 \\
\hline Bicoronal & $19(10.6)$ & $14(7.8)$ & $5(9.8)$ & 0.09 \\
\hline Unilambdoid & $4(2.2)$ & $2(1.6)$ & $2(3.9)$ & 0.11 \\
\hline Multisuture & $7(3.9)$ & $5(3.9)$ & $2(3.9)$ & 0.99 \\
\hline \multicolumn{5}{|l|}{ Surgeon identities } \\
\hline Neurosurgeon 1/Plastic Surgeon 1 & & 2 & 3 & \\
\hline Neurosurgeon 1/Plastic Surgeon 2 & & 40 & 3 & \\
\hline Neurosurgeon 2/Plastic Surgeon 1 & & 16 & 8 & \\
\hline Neurosurgeon 2/Plastic Surgeon 2 & & 26 & 5 & \\
\hline Neurosurgeon 3/Plastic Surgeon 1 & & 11 & 25 & \\
\hline Neurosurgeon 3/Plastic Surgeon 2 & & 33 & 7 & \\
\hline
\end{tabular}

tively, all JPDs were placed to closed, continuous suction drainage. The number of depressions of the attached bulb was not recorded. The decision to remove JPDs was also made by the treating surgeons on an individual basis, and all JPD-group patients had them in place at the study's end point. All patients were admitted to the ICU postoperatively, where intake and outputs were strictly monitored. No strict transfusion thresholds were predetermined in this study, so patients received blood product transfusions as clinically indicated according to the judgment of the treating surgeon and/or intensive care physician.

\section{Laboratory Values and Length of Stay}

Patients' electronic medical records were queried for laboratory values for 3 periods. "Preoperative," "immediate postoperative," and "late postoperative" data points were defined as those collected during the periods 7-14 days prior to surgery, the 6 hours within conclusion of surgery, and 24-48 hours after surgery, respectively. Laboratory values examined included hemoglobin, hematocrit, and serum sodium. Estimated blood loss (EBL) and intraoperative and perioperative (within 24 hours of surgery) transfusion data were obtained from anesthesia and ICU records. When JPDs were present, their output was tracked over the time while they were in place.

Patient records were examined for length of stay in the ICU and the hospital. In addition, all records of study patients were examined for postoperative visits to the emergency department, clinic, or hospital. These records were then analyzed for evidence of postoperative wound concerns, including suspicion for infection or dehiscence, or whether any further operative intervention was required.

\section{Statistical Analysis}

Data were collected and processed in a computerized spreadsheet program (Microsoft Excel) and Stata statistical software (StataCorp) was used for analysis. Data across treatment groups were compared using a 2-tailed unpaired Student's t-test and parametric regression models, as appropriate.

\section{Results \\ Patient Population and Intraoperative Changes}

Key pre- and postoperative characteristics are shown in Table 1. There was no difference in age or craniosynostosis type between the two groups. Sagittal and metopic synostosis were the most commonly treated conditions overall. Each of the 3 neurosurgeons was involved in 48, 55, and 76 cases and each of 2 plastic surgeons was involved in 65 and 114 cases, respectively. The decision to place a JPD was strongly associated with the identities of the operating plastic surgeon, but not the neurosurgeon. When Plastic Surgeon 1 participated in the case, there were more patients without than with a JPD (36 vs 29), whereas the reverse was true when Plastic Surgeon 2 participated in the surgery (99 patients with a JPD, 15 without). Overall, more patients received a JPD $(n=128[71.5 \%])$ than did not.

Intraoperative changes were determined by comparing data from the immediate postoperative period to the preoperative assessments, stratified by those receiving and not receiving a JPD (Table 2). The mean duration of the operation was $140 \pm 40$ minutes. The mean EBL for all patients was $134.0 \pm 94.1 \mathrm{ml}: 139.8 \pm 105.3 \mathrm{ml}$ in the JPD group and $128.3 \pm 57.0 \mathrm{ml}$ in the no-JPD group (a nonsignificant difference). Notably, patients in the JPD group received more transfusion intraoperatively (88.1 vs 56.2 $\mathrm{ml})$. The underlying cause of this difference is not clear, and it did not co-segregate with surgeon identity. Even so, 
TABLE 2. Perioperative outcome data

\begin{tabular}{|c|c|c|c|}
\hline \multirow[b]{2}{*}{ Factor } & \multicolumn{2}{|c|}{ Group (\%)* } & \multirow{2}{*}{$\begin{array}{c}\mathrm{p} \\
\text { Value }\end{array}$} \\
\hline & JPD & No JPD & \\
\hline No. of patients & 128 & 51 & \\
\hline \multicolumn{4}{|l|}{$\begin{array}{l}\text { Values btwn preop to immediate } \\
\text { postop periods }\end{array}$} \\
\hline Length of op (mins) & $153 \pm 40$ & $133 \pm 38$ & 0.33 \\
\hline $\mathrm{EBL}(\mathrm{ml})$ & $139 \pm 105$ & $128 \pm 57$ & 0.46 \\
\hline Blood transfusion (ml) & $88.1 \pm 87.0$ & $56.2 \pm 85$ & 0.07 \\
\hline $\mathrm{Hg}$ changes $(\mathrm{g} / \mathrm{dl})$ & $-0.32 \pm 2.14$ & $-0.35 \pm 2.21$ & 0.39 \\
\hline Hct changes (\%) & $-1.00 \pm 6.16$ & $-1.09 \pm 6.45$ & 0.21 \\
\hline Sodium changes (mEq/L) & $-0.47 \pm 3.03$ & $-0.77 \pm 2.43$ & 0.19 \\
\hline \multicolumn{4}{|l|}{$\begin{array}{l}\text { Values for } 48 \text {-hr period after } \\
\text { the op }\end{array}$} \\
\hline JPD output (ml) & $222 \pm 142$ & NA & \\
\hline Hg changes (g/dl) & $-0.79 \pm 2.00$ & $-1.07 \pm 1.82$ & 0.32 \\
\hline Hct changes (\%) & $-2.76 \pm 5.53$ & $-3.29 \pm 4.73$ & 0.42 \\
\hline Sodium changes $(\mathrm{mEq} / \mathrm{L})$ & $-2.90 \pm 3.44$ & $-1.80 \pm 3.07$ & 0.06 \\
\hline $\begin{array}{l}\text { No. of patients w/ sodium } \\
<135 \mathrm{mEq} / \mathrm{L}(\%)\end{array}$ & $47(36.7)$ & $14(27.3)$ & 0.24 \\
\hline \multicolumn{4}{|l|}{ Other outcome data } \\
\hline ICU LOS (days) & $1.60 \pm 1.33$ & $1.56 \pm 1.05$ & 0.84 \\
\hline Hospital LOS (days) & $3.46 \pm 1.56$ & $3.55 \pm 1.08$ & 0.70 \\
\hline ED visits in 60 days (\%) & $8(6.3)$ & $5(9.8)$ & 0.56 \\
\hline
\end{tabular}

$\mathrm{ED}=$ emergency department; $\mathrm{Hct}=$ hematocrit; $\mathrm{Hg}=$ hemoglobin; $\mathrm{LOS}=$ length of stay; NA = not applicable.

Values are presented as the mean \pm SD or number (\%).

* Negative values signify a decrease over time.

both groups exhibited comparable small changes in hemoglobin (a drop of $<0.5 \mathrm{~g} / \mathrm{dl}$ ), hematocrit (mean decrease of $1 \%$ ), and serum sodium (mean change of $0.55 \mathrm{mEq} / \mathrm{L}$ ) at the conclusion of the surgery (Table 2).

\section{JPD Usage, Late Postoperative Changes, and Follow-Up}

The JPD was removed on postoperative day (POD) 1 in 93 cases, POD 2 in 29, and POD 3 in 5. The average output of the JPD was $215 \pm 131 \mathrm{ml}$. Laboratory values obtained 24 hours after the conclusion of surgery were compared with those obtained in the immediate postoperative period (Table 2). For both groups, there were significant differences in these sets of values. For the JPD and no-JPD groups, hematocrits dropped by means of 2.76 and 3.29 points, and serum sodium by means of 2.9 and $1.8 \mathrm{mEq} / \mathrm{L}$, respectively. Using a serum sodium of $135 \mathrm{mEq} / \mathrm{L}$ as a cutoff, nearly one-third of the patients had hyponatremia. However, no neurological or renal consequences were associated with this. There were also no records of bradycardia associated with the presence of a JPD.

Finally, Table 2 displays data on the mean lengths of ICU and total hospital stay for patients in both groups. These values did not differ significantly between the groups. No neurological complications were noted in our series at the time of last follow-up, and all patients were discharged to home. During the 60-day period after discharge, 13 patients made emergency department visits. Of the 13 visits, most of which were related to fever and vom- iting, 4 patients had documented wound-related concerns. One patient in the JPD group fell out of his crib while in the hospital. He was discharged to home on POD 5 but then returned with a CSF leak through the primary cranial incision; he was taken to the operating room for wound revision, and the leak did not recur by final follow-up. One patient presented with parental concern of wound swelling but no treatment was initiated. One patient in the no-JPD group presented with fluid leak from the primary cranial incision and was oversewn in the emergency department, and another patient, also in the no-JPD group, presented with minor wound erythema and received a 5-day course of oral antibiotics at the discretion of the on-call surgeon. There were no wound infections noted in either group postoperatively.

\section{Discussion}

\section{Rationale and Limitations of the Study}

The use of JPDs in pediatric CFRS is common, but there is little evidence for or against this practice. This retrospective study was initiated to obtain preliminary information on the positive and negative effects associated with JPD usage in CFRS. If the findings were significant, a prospective trial could be initiated. Because this is a prestudy, we were willing to accept significant limitations of our methodology. A point of contention among CFRS surgeons (and indeed among all surgeons using JPDs) is whether these drains have an increased risk of postoperative complications. This study attempts to address the potentially serious complications of JPD placement independent of the intraoperative technique or decision-making process that led to placement of a JPD. Nevertheless, several caveats are important to address.

The most obvious and important shortcoming is that even though the number of patients in our population was not small, once they were stratified by surgeon identities, the number in each silo quickly dwindled. Importantly, there were no criteria used here for the placement or removal of JPDs; these were left to the discretion of the treating surgeon. This is important because every surgeon is different, from surgical site preparation, to incisions, extent of craniectomy, types of reconstruction, length of the surgery, and the decisions to transfuse and use JPDs. These decisions are hard to quantify but could directly affect outcomes. Furthermore, it would make clinical sense to stratify craniosynostosis types together with surgeon identities to minimize selection bias, but this was not done because of sample size limitations. We suspect that most of the differences in operating time, EBL, and blood transfusion can be explained by the personnel involved, even though we may lack the statistical power to confirm or reject the hypothesis. A power analysis was not performed given the retrospective nature of the study and the lack of previous data to suggest a minimum detectable difference between the two groups. Even so, we sought to demonstrate that, at the conclusion of all included operations, the two groups were similar in terms of laboratory characteristics.

\section{Changes in Hemoglobin, Hematocrit, and Serum Sodium}

As expected, there were significant decreases in hemo- 
globin and hematocrit during the first 24-hour period after surgery. These decreases have been found in other studies as well. In both the JPD and no-JPD groups, hemoglobin and hematocrit decreased on average, albeit with an error margin that spanned into the positive. The possibility of fluid shift between inter- and intravascular spaces and into the subgaleal space, and overhydration or dehydration, probably all contributed to these variations. Despite these limitations, the use of a JPD does not appear to be associated with the postoperative changes in hemoglobin/ hematocrit.

The decrease in serum sodium was greater in the JPD than the no-JPD group, showing a trend toward significance but not ultimately reaching a statistically significant level. Overall, we found that a substantial number of our pediatric CFRS patients were at risk for postoperative hyponatremia, but there was no convincing evidence that EBL or JPD usage contributed to a drop in serum sodium at the immediate and 24-hour postoperative time points. The finding of new postoperative hyponatremia has been consistently made in several CFRS series, but the cause has been disputed. We expected that intravascular volume depletion in our patients would lead to appropriate release of antidiuretic hormone (ADH), causing hemodilution and low serum sodium. Indeed, this was the theorized cause of hyponatremia in a study by Cladis et al., ${ }^{2}$ the largest retrospective CFRS series to date (JPD status was not studied). In that study, patients in whom hyponatremia developed had greater mean intraoperative blood loss than those without hyponatremia, which the authors felt was consistent with appropriate ADH release as an etiology. However, many patients in their series received hypotonic intravenous fluids postoperatively, and other studies have failed to identify a postoperative serum ADH level increase in pediatric CFRS patients. ${ }^{5,9}$

\section{Hospital Course and Delayed Wound Complications}

Some CFRS surgeons avoid using JPDs for fear that hospital stay could be prolonged while they await JPD output to drop to an appropriately low level or for JPD fluid to change to a nonbloody consistency. Indeed, little in the literature has examined the appropriate length of time for surgical drains to remain in place (and no studies have examined this concept in conjunction with CFRS), although studies in the orthopedic literature suggest that the optimal time for removal is 24 hours postoperatively. ${ }^{3}$ We did not expect that the use of JPDs would lengthen the hospital course, and our results failed to demonstrate significant effect on length of stay in either the ICU or the hospital.

We also examined records for evidence of delayed postoperative wound complications, as evidenced by presentation to the hospital (for inpatient admission) or to the emergency department. While this has not been examined in CFRS series in particular, studies across multiple specialties have failed to yield convincing evidence for a link between surgical drains and wound complications or the appropriateness of prophylactic antibiotics while drains are in place., ${ }^{711}$ In our series, one patient in the JPD group required operative revision of wounds, and the number of patients presenting with wound-related complaints was not significantly different between the groups, although in view of the small effect size in this population, a larger number of patients would be needed to capture a true intergroup difference.

\section{Future Studies}

This was a prestudy meant to serve as a precedent for future prospective work regarding the use of JPDs in pediatric CFRS. Accounting for the aforementioned limitations, we were unable to detect an association between JPDs and our outcome measures in this series. Future retrospective studies could further minimize selection bias by focusing on more defined cohorts-for example, JPD and no-JPD groups in patients who underwent a particular reconstructive procedure such as frontoorbital advancement or sagittal synostectomy. Other specific cohorts could include patients who had a certain range of operative times or those who underwent surgery with a certain surgical team. Furthermore, our data suggest that the effect size examined here may be small and that future studies should increase the sample size (e.g., by examining data across multiple institutions). Finally, controlling for variables such as perioperative blood transfusion volumes, weight (i.e., estimated blood volume), and preoperative hemoglobin would be ideal but was not possible given our sample size limitations.

\section{Conclusions}

Pediatric patients undergoing CFRS are at risk for serious perioperative morbidity from blood loss, fluid shifts, and wound complications, making the use of JPDs in this population worthy of exploration. We were able to duplicate the high rate of postoperative hyponatremia seen in other studies, but we found no convincing evidence of complications as a result of JPD placement with regard to perioperative blood loss, hyponatremia, length of stay, or wound complications requiring operative intervention. There are limitations in studying multiple surgeons' decisions and results retrospectively. To our knowledge, however, this is the first larger series of JPD usage in the literature, and the lack of an effect suggested here should inform future retrospective and possibly prospective studies in this area. Our study indicates that the use of JPDs in CFRS is probably safe, though the benefits are still unclear, and that all these patients should still be monitored very carefully for postoperative complications.

\section{References}

1. Choi SY, Yoon SM, Yoo CJ, Park CW, Kim YB, Kim WK: Necessity of surgical site closed suction drain for pterional craniotomy. J Cerebrovasc Endovasc Neurosurg 17:194202, 2015

2. Cladis FP, Bykowski M, Schmitt E, Naran S, Moritz ML, Cray J, et al: Postoperative hyponatremia following calvarial vault remodeling in craniosynostosis. Paediatr Anaesth 21:1020-1025, 2011

3. Drinkwater CJ, Neil MJ: Optimal timing of wound drain removal following total joint arthroplasty. J Arthroplasty 10:185-189, 1995

4. Hernández-Palazón J, Tortosa JA, Sánchez-Bautista S, Martínez-Lage JF, Pérez-Flores D: Cardiovascular disturbances 
caused by extradural negative pressure drainage systems after intracranial surgery. Br J Anaesth 80:599-601, 1998

5. Hudgins RJ, Burstein FD, Boydston WR: Total calvarial reconstruction for sagittal synostosis in older infants and children. J Neurosurg 78:199-204, 1993

6. Karamchandani K, Chouhan RS, Bithal PK, Dash HH: Severe bradycardia and hypotension after connecting negative pressure to the subgaleal drain during craniotomy closure. $\mathbf{B r}$ J Anaesth 96:608-610, 2006

7. Kosins AM, Scholz T, Cetinkaya M, Evans GR: Evidencebased value of subcutaneous surgical wound drainage: the largest systematic review and meta-analysis. Plast Reconstr Surg 132:443-450, 2013

8. Lavoie J: Blood transfusion risks and alternative strategies in pediatric patients. Paediatr Anaesth 21:14-24, 2011

9. Levine JP, Stelnicki E, Weiner HL, Bradley JP, McCarthy JG: Hyponatremia in the postoperative craniofacial pediatric patient population: a connection to cerebral salt wasting syndrome and management of the disorder. Plast Reconstr Surg 108:1501-1508, 2001

10. Nguyen TT, Lam HV, Phillips M, Edwards C, Austin TM: Intraoperative optimization to decrease postoperative PRBC transfusion in children undergoing craniofacial reconstruction. Paediatr Anaesth 25:294-300, 2015

11. Petrowsky H, Demartines N, Rousson V, Clavien PA: Evidence-based value of prophylactic drainage in gastroin- testinal surgery: a systematic review and meta-analyses. Ann Surg 240:1074-1085, 2004

12. Van Roost D, Thees C, Brenke C, Oppel F, Winkler PA, Schramm J: Pseudohypoxic brain swelling: a newly defined complication after uneventful brain surgery, probably related to suction drainage. Neurosurgery 53:1315-1327, 2003

\section{Disclosures}

The authors report no conflict of interest concerning the materials or methods used in this study or the findings specified in this paper.

\section{Author Contributions}

Conception and design: Vasudevan. Acquisition of data: Chern, Vasudevan. Analysis and interpretation of data: Chern, Vasudevan, Oh, Garcia. Drafting the article: Vasudevan, Oh, Tubbs, Garcia, Reisner. Critically revising the article: all authors. Reviewed submitted version of manuscript: Chern, Vasudevan, Tubbs, Reisner. Statistical analysis: Vasudevan.

\section{Correspondence}

Joshua J. Chern, Children's Healthcare of Atlanta, Neurosurgery, 5455 Meridian Mark Rd. NE, Ste. 540, Atlanta, GA 30342. email: joshua.chern@choa.org. 